\title{
Simplification of the landscape under high energy milling of molecular solids exhibiting polymorphism.
}

\author{
J. Linol and G. Coquerel \\ Unité de Croissance Cristalline et de Modélisation Moléculaire, SMS \\ UPRES EA3233 -IRCOF, Université de Rouen \\ F-76821 Mont Saint Aignan Cedex France \\ gerard.coquerel@univ-rouen.fr
}

\begin{abstract}
:
Polymorphism is of high occurrence among molecular solids quite often the number of polymorphs exceeds 3 4.Several studies have shown that under high energy milling if $\mathrm{Tg}<$ Tmilling there is a simplification of the landscape. That is to say for a given milling intensity, whatever the number of polymorphs existing without mechanical stress, a single polymorph corresponds to the steady state. By changing the milling intensity or the composition of the medium (wet milling for instance) a different variety might be accessible. This solid phase can be: the stable thermodynamic form, a metastable thermodynamic form, a dynamic new metastable form, a solvate, a co-crystal, etc. It is only under special conditions of intensity and composition that two polymorphs can co-exist as dual inter-converting steady states.
\end{abstract}

Keywords: High Energy Milling, polymorphism, Steady State

\section{INTRODUCTION}

In many areas of material science as well as in pharmaceutical industry, the understanding and control of polymorphism are two essential issues [1],[2].The polymorphism is the ability of the same chemical substance to exist in different crystalline forms. The access to a pure and stable polymorph can be a difficult task because of the narrow energy gap between the varieties i.e. a very small $\Delta G$ separates the free enthalpies of the solid phases.

The High Energy Milling (HEM) is a well known route to reach polymorphic forms, amorphous state, co-crystals or solvates, host-guest inclusions of molecular compounds [3],[4],[5].

In this paper, the polymorphic transitions of two molecular compounds under high energy milling are studied. Several studies have shown that under high energy milling if the temperature of glass transition $\mathrm{Tg}<\mathrm{T}_{\text {milling }}$ there the steady state is likely to be a defective crystal lattice. Moreover, for a given milling intensity I, whatever the number of polymorphs existing without mechanical stress, a single defective polymorph corresponding to the steady state is usually observed. By changing the milling intensity or the composition of the medium (wet milling for instance) a different variety might be accessible. This solid phase can be (figure 1):

- The stable thermodynamic form: This is the case of the 5-methyl-5-(4'-methylphenyl) hydantoïne: this molecule exhibits two polymorphs and under 'wet' milling, the kinetic of conversion from metastable form to stable form is more than 40 times faster than in a stirred solution [6] (Figure 1: case(a))

- A metastable thermodynamic form: in this case, an inversion of the relative stability between two polymorphic forms is possible. For example, $Г$-form of sorbitol, which is the most stable form and the most common polymorph, converge into the metastable A-form, under high energy milling (dry milling conditions)[7].

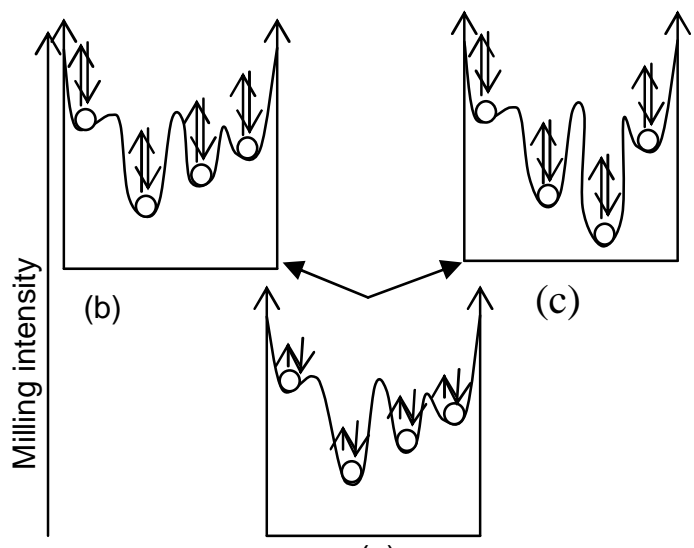

(a)

Figure 1 Case (a): Thermodynamic conditions $(I=0)$, case (b): I $>0$, No inversion of the relative stability: the steady state is the thermodynamic stable form. case (c): I $>0$, Inversion of the relative stability: the steady state is one of the metastable forms.

- A mixture of two polymorphs as also been described but it is a rare case: e.g. sulfathiazole, which exhibits three polymorphic forms. At the high milling intensity, two polymorphic forms coexist: the milling initiate the transition from stable form III into metastable form I via an intermediate amorphous state. However, during the milling, energy was accumulated in the sample and was able to induce a fast conversion back to form III [8]

The milling conditions (for example the presence of a medium) influence the nature of the steady state. In the next section, the parameters, which control the nature of the steady state, will be described.

This is an Open Access article distributed under the terms of the Creative Commons Attribution-Noncommercial License (http://creativecommons.org/licenses/by-nc/3.0/), which permits unrestricted use, distribution, and reproduction in any noncommercial medium, provided the original work is properly cited. 


\section{THEORY OF MILLING}

Under external forces (e.g. irradiation or HEM), dynamic equilibrium can be far from thermodynamic equilibrium. The stressed materials undergo two opposite processes: damage and recovery. When a dynamic equilibrium between damage and recovery is reached, a steady state is observed. That is to say the nature and the spatial distribution of the solid phase(s) do not evolve.

The nature of the steady state depends on several parameters [9]:

- The composition of the system: In our case, it was the presence (or not) of solvent(s) but for instance it could be the composition in a second solute or in an excipient: i.e. co-grinding [10].

- Mean milling temperature: Under HEM, two types of temperature exist:

- The "local" temperature is the sudden rise in temperature during a shock. The peak temperature is difficult to measure directly because of the microscopic and dynamic nature of the process [11]

- The mean milling temperature corresponds to the rise in temperature inside the vial and could be monitored by a system of vial equipped with a thermal probe. The mean temperature is a function of the shock energy and the impact frequency.

Two cases could be differentiated according to the mean milling temperature:

$\circ$ If the mean milling temperature < Temperature of glass transition: an amorphous state is likely to be obtained.

- If the mean milling temperature > Temperature of glass transition: a defective crystalline phase is likely to be obtained.

\section{- Milling intensity}

The milling intensity is defined as the momentum transferred by a ball to the unit mass of powder per unit time. The formula (Equation1) of milling intensity is:

$\mathrm{I}=(m b$ *Vb*f) / mp

Equation (1): Milling Intensity as a function of $\mathrm{mb}$ (total mass of the balls), $\mathrm{Vb}$ (maximum velocity of balls), $f$ (impact frequency) and $\mathrm{mp}$ (mass of the powder submitted to HEM)

In so called 'local model' the milling intensity is applied to the volume of trapped powder [12]. According to the literature [8], in the case of planetary milling, the impact velocity and the shock frequency depend on the disk radius and the milling couple $(\Omega, \omega)[\Omega=$ velocity of disk, $\omega=$ velocity of vial]. If these parameters are constant, the milling intensity depends solely on the ratio $\mathrm{R}=\mathrm{mb} / \mathrm{mp}$

\section{EXPERIMENTAL}

The planetary mill used is the Pulverisette 4 (P4) from Fritsch (Oberstein, Germany). This mill is composed of two vials $(80 \mathrm{ml})$ attached to a horizontal disk, the vial rotation speed and the disk rotation speed being independent. The material used is the tungsten carbide (density $=14,7$ ).

\section{RESULTS AND DISCUSSION}

The behaviours of two pharmaceutical compounds, under high energy milling, have been investigated: The $( \pm)$ modafinil and the compound $A$.

These compounds exhibit a temperature of glass transition below the mean milling temperature: that is to say, the steady state should be a crystalline phase.

\section{- $\quad 4.1( \pm)$ modafinil}

$( \pm)$ modafinil exhibits seven polymorphic forms. At $P=1$ atm, whatever the temperature, Form I is the stable form and form III the second best in term of stability, but the difference in energy is quite small. Without particular precaution, form I and III are concomitant polymorphs [13]. At the moderate milling intensity (charge ratio $R=15$ ), all the polymorphic forms converge into the defective form III. Under HEM, a simplification of the landscape and an inversion of the relative stability between Form I and Form III have been observed [14].

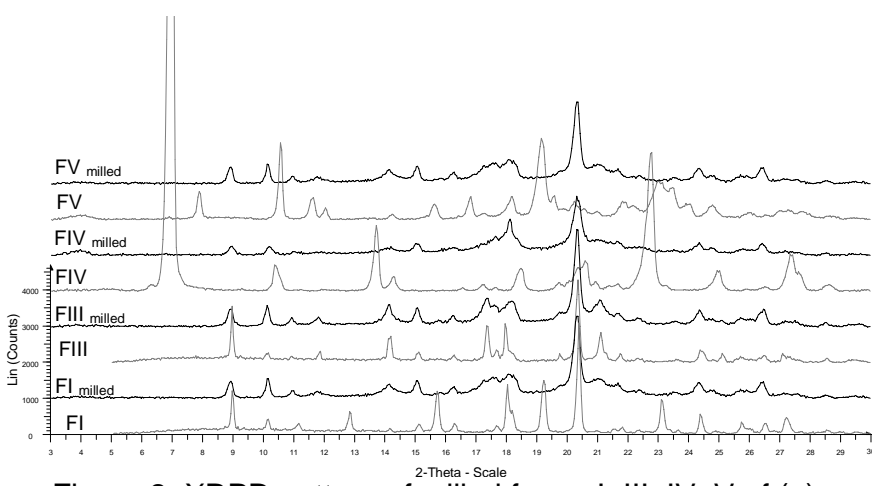

Figure 2: XRPD pattern of milled forms I, III, IV, V of $( \pm)$ modafinil.

The addition of the solvent (water) influences the nature of the steady state:

- With $3 \%$ of water, all polymorphic forms converge into a defective form I. Therefore, the nature of the stable thermodynamic phase remains unchanged under wet milling conditions.

It is important to note that the solubility of the racemic compound -form $\mathrm{I}$ - is ca. $0.02 \%$ in water at room temperature. Therefore it is likely that the combination of shock and friction effects is modified.

o Between $1 \%$ and $3 \%$ and whatever the milling duration, a mixture between form I and form III was reached as the steady state. Alike sulfathiazole (see introduction section) a competition between two opposite processes occurs: the one favours Form I ('wet' milling) and the other favours form III ('dry' milling). 
The contrasted results obtained by using 'dry' and 'wet' millings prompted us to design a cyclic process schematized in figure 3 [14].

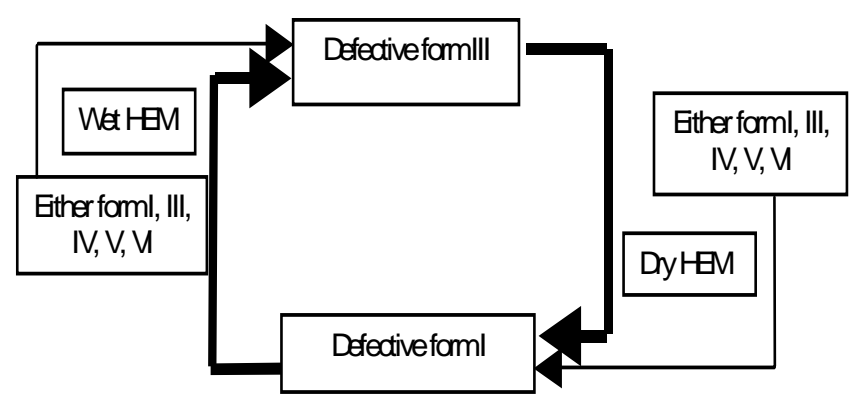

Figure 3: Alternate crystallization of form I and form III under consecutive dry and wet HEM.

Whatever the milling conditions, a simplification of the landscape was observed. However, the presence of the solvent influences the nature of the steady state: form I and/or form III.

\section{- $\quad 4.2$ Compound A}

The second compound studied (a patent has just been filed) exhibits seven polymorphic forms. At $\mathrm{P}=1 \mathrm{~atm}$, and $\mathrm{I}=$ 0 whatever the temperature, Form II is the stable form. HEM led for the first time to a new polymorph: Form V. At a moderate milling intensity, all the polymorphic forms converge into this new variety. Therefore, an inversion of the relative stability between form II and form V, under mechanical stress, has been obtained. The resolution of the crystal structure of this new polymorph has shown that the mechanism of transition, during HEM, is of destructive / reconstructive type.

Without mechanical stress, the form $V$ span of life depends on the energy of the formation process (table 1 and figure 4).

\begin{tabular}{|c|c|c|c|c|}
\hline Process & (a) & (b) & (c) & \multicolumn{1}{c|}{$(\mathrm{d})$} \\
\hline Life time & $\begin{array}{l}<15 \\
\text { days }\end{array}$ & 2months & $\begin{array}{l}6 \\
\text { months }\end{array}$ & $\begin{array}{l}>14 \\
\text { months }\end{array}$ \\
\hline
\end{tabular}

Table 1: Life time of form $V$ according to the process: (a)

HEM I = (b) Medium EM (c) Seeding super-cooled molten

state by freshly prepared form $\mathrm{V}$ by process $-\mathrm{b}$ - and (d) Spray drying.

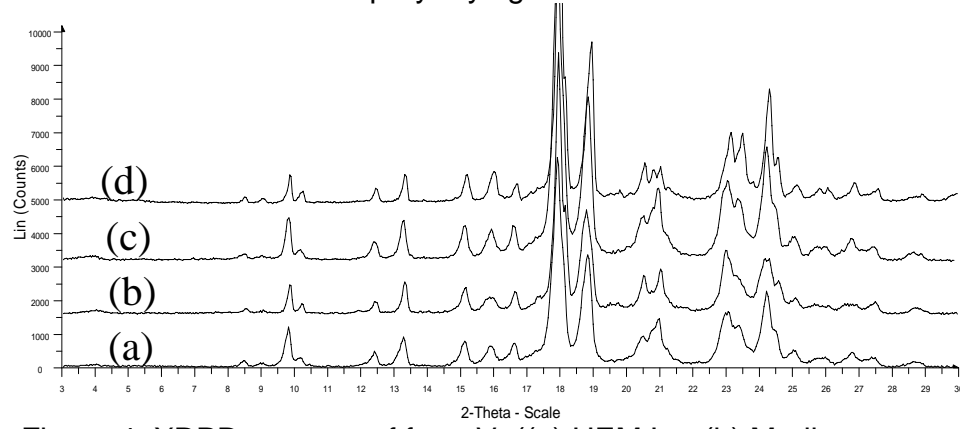

Figure 4: XRPD patterns of form V: ((a) HEM I = (b) Medium EM (c) Seeding super-cooled molten state by freshly prepared form $\mathrm{V}$ by process -b- and (d) Spray drying.

The form $V$ obtained by spray-drying exhibits a gap in crystallinity and consistently a longer life time than form $V$ obtained by milling or seeding of the molten state by freshly prepared seeds of form $\mathrm{V}$ (process $\mathrm{b}$ in table 1 ).

Based on that result it is high probable that the kinetics of the return to form II can be speed-up by wet milling so that form II and form could be in rapid inter-conversion in a dynamical steady state.

\section{CONCLUSION}

Whatever the number of polymorphs existing at $\mathrm{I}=0$, a single defective solid phase is usually obtained for a milling intensity I above a given threshold and a temperature above the temperature of glass transition. Therefore most of the time, dry HEM leads to a sharp simplification of the landscape. This can be conceived as resulting from the high energy shock wave spreading through the solid phase and preventing them to remain in any bottom of energy well except the one of lowest dynamical energy. The rare exception to that rule seems to be due to a high kinetics of recovery. This is why wet milling with a precise quantity -and small- quantity of solvent could be a general method to form a dual steady state between damage and recovery with two co-existing polymorphs.

If the steady state is different from the thermodynamic phase, on return to $\mathrm{I}=0$ the relaxation of this frozen state (far thermodynamic equilibrium) induces a solid-solid transition to the stable form. This study suggests that the lower the crystallinity of the steady state the faster the return to the stable phase.

[1] Hilfiker R., 2006, Polymorphism in the Pharmacutical Industry, Wiley-VCH, Weinheim.

[2] Brittain H. G., 1999, Polymorphism in Pharmaceutical solids, Marcel Dekker, New York

[3] Shaktshneider T.P, Boldyrev V.V., 1999, Reactivity of molecular solid: Mechanical synthesis and mechanical activation of drugs, Boldyreva E., Boldyrev V.V Editions, Wiley Chichester, 271-311.

[4] Shaktshneider T.P, Danède F., Capet F., Willart J.F., Descamps M., Myz S.A., Boldyreva E., Boldyrev V.V., 2007, Grinding of drugs with pharmaceutical excipients at cryogenic temperatures, .J. therm. Analysis and calorimetry, 89, 699707.

[5]Willart J.F., Caron V., Lefort R., Danède F., Prevost D., Descamps M., Athermal character of the solid state amorphous of lactose induced by ball milling, 2004, Solid state comm., 132, 693-696.

[6] Linol J, Coquerel G, Influence of high energy milling on the kinetics of the polymorphic transition from the monoclinic form to the orthorhombic form of: ( \pm )5-methyl-5-(4'methylphenyl)hydantoïne, 2007, .J. therm. Analysis and calorimetry,, 90, 367-370.

[7]Willart J.F., Lefebvre J., Danède F., Comini S., Looten P., Descamps M., polymorphic transformation of the form $\Gamma$-form of D-sorbitol upon milling: structural and nanostructural analyses, Solid State Communications, 2005, 135, 519-524.

[8]Shaktshneider T.P, Boldyrev V.V., Reactivity of molecular solid: Mechanical synthesis and mechanical activation of drugs, 1999, E. Boldyreva, V.V Boldyrev Editions, Wiley Chichester, 271-311.

[9]Abdellaoui M., Gaffet E., The physics of mechanical alloying in a planetary ball mill: mathematical treatment, 1995, Acta metall. Mater, 43, 1087-1098.

[10]Descamps M., Willart J.F., Dudognon E., Caron V., Transformation of pharmaceutical compounds upon milling and comilling: The role of Tg, 2006, J. Pharm. sci, 96, 13981407. 
[11]Schwarz B., Koch C.C, Formation of amorphous alloys by the mechanical alloying of crystalline powders of pure metals and powders of intermetallics, 1986, apply. Phys. Lett., 49, 146.

[12]Begin-Colin S., Girot T., Le Caër G., Mocellin A., Kinetics and mechanisms of phase transformations induced by ball milling in antase $\mathrm{TiO}_{2}$, 2000, Solid State Chem., 149, 41-48.

[13] Bernstein, J., Davey, RJ., Henck, JO., Concomittent polymorphs, Angew. Chem. Int. Ed., 38(1999), 3440.

[14] Linol J., Morelli T., Petit M.N., Coquerel G., Inversion of the relative stability between two polymorphic forms of $( \pm)$ modafinil under dry high energy milling: comparisons with results obtained under wet high energy milling, 2007, Cryst. Growth and Design, 7, 1608-1611. 